\title{
ACERCAMIENTO AL ACCIONAR ÉTICO-MORAL DEL CIENTÍFICO QUE TRABAJA CON ANIMALES DE EXPERIMENTACIÓN
}

\author{
Ángel R. Concepción Alfonso*, R. de la Peña Pino** y J. García Capote ${ }^{* * *}$
}

Resumen: El avance vertiginoso de la revolución científico-técnica y el uso no siempre racional y ético de animales en la investigación han conducido a muchos investigadores a preocuparse por el destino de las especies utilizadas, lo que ha generado numerosos estudios en las últimas décadas. Se han formulado múltiples reglamentaciones y se ha pensado acerca de la aplicación de principios éticos en las investigaciones.

Este artículo considera la utilización de métodos alternativos a la investigación con animales, siempre que sea posible; revisa el principio de las tres $\mathrm{R}$ y otros aspectos relacionados, y discute sobre las responsabilidades del científico con el producto final de sus resultados.

Palabras clave: bioética del investigador, animales de laboratorio

\section{APPROACHING TO ETHICAL-MORAL THINKING OF SCIENTISTS WORKING WITH EXPERIMENTAL ANIMALS}

Abstract: The vertiginous advance of the scientific technical revolution and the use not always rational and ethical of animals in research, has provoked that many researchers worry about the future of animal species used, which has been the topic of many studies in the last decade. Multiple regulations have been formulated and the application of ethical principles in research has been considered.

This paper considers the use of alternative methods in research with animals whenever possible, reviews the principle of the three Rs and other related aspects and reflect on the responsibilities of the scientist with the end product of his/her results.

Key words: bioethics of the researcher, laboratory animals

\section{REFLEXÔES SOBRE O AGIR ÉTICO-MORAL DO CIENTISTA QUE TRABALHA COM ANIMAIS DE PESQUISA}

Resumo: O avanço vertiginoso da revolução científico-técnica e o uso nem sempre racional e ético dos animais na pesquisa, levaram muitos pesquisadors a se preocupar com o destino das espécies utilizadas, o que gerou numerosos estudos nas útlimas décadas. Formularam-se inúmeras regulamentações e reflexões sobre a aplicação de princípios éticos nestas pesquisas. Este artigo considera a utilização de métodos alternativos na pesquisa com animais, sempre que seja possível, revisa o princípio dos três $\mathrm{R}$ e outros aspectos relacionados, bem como discute sobre as responsabilidades do cientista com o produto final de seus resultados.

Palavras chave: bioética da pesquisa, animais de laboratório

* Jefe del grupo Biomodelos, Investigador Auxiliar, Centro Nacional de Genética Médica. Instituto Superior de Ciencias Médicas de La Habana, Cuba

** Investigador Centro Nacional de Genética Médica. ISCMH, Cuba

*** Profesora Titular-Consultante de Filosofía del ICBP "Victoria de Girón". ISCMH, Cuba

Correspondencia: aconce@giron.sld.cu 


\section{Introducción}

La experimentación con animales ha permitido un desarrollo cada vez más acelerado de los conocimientos biológicos y del bienestar del hombre y de los propios animales. Por ello el destino de estas especies ha comenzado a despertar un gran interés, $\mathrm{y}$ evitar o disminuir su sufrimiento ha sido objeto de numerosos estudios en las últimas décadas. Esto ha llevado al establecimiento de múltiples reglamentaciones $\mathrm{y}$, por otro lado, ha hecho pensar en las relaciones con el medio ambiente, el cual se ve afectado(1).

Las ciencias biomédicas y otras afines avanzan rápidamente con la utilización de modelos animales adecuados, que posibilitan la evaluación de nuevos medicamentos para el tratamiento y prevención de diferentes enfermedades. También, es éticamente reconocido que no se deben emplear nuevos medicamentos, sustancias, ni dispositivos en seres humanos a menos que las pruebas previamente efectuadas en animales permitan hacer una suposición razonable de su inocuidad. En efecto, los editores de las principales revistas biomédicas exigen que los investigadores declaren haber seguido las normas de la propia institución y las leyes nacionales sobre el cuidado y uso de animales.

Por tanto, el empleo de animales de experimentación para predecir los probables efectos de ciertos procedimientos que se aplicarían en el ser humano implica responsabilidad por el bienestar animal(2). En ocasiones es incluso más ético y razonable el uso de métodos alternativos(3).

Partiendo de estas consideraciones, el presente trabajo tiene como objetivos:

- Analizar la importancia de la aplicación de principios éticos en las investigaciones con diferentes modelos experimentales y su estrecha vinculación con el medio ambiente.

- Reflexionar sobre los cuidados y consideraciones que debemos cumplir, en dependencia del tipo de experimento y del animal de elección.

- Argumentar sobre la utilización de métodos alternativos, siempre que sea posible, en las investigaciones con animales de experimentación, así como respecto de la responsabilidad del científico con el producto final del trabajo.

\section{La valoración bioética respecto al uso de animales en la experimentación}

La valoración ética supone el establecimiento básico de un grupo de principios(4). En efecto, los principios y paradigmas utilizados en la ética médica humana pueden servir para aplicarlos en las investigaciones con animales. El concepto de "paradigma" significa una "empresa científica en el entramado social, donde están presentes no sólo las teorías, sino, también, el abanico de creencias, actitudes, procedimientos, técnicas, valores, etc."(5).

La ética antropocentrista descansa en la idea de que la vida humana es mucho más valiosa que la vida animal, noción que subyace en muchas actitudes y conductas habituales. Esta noción, llevada al extremo, induce a creer que la vida humana es la única que encierra un valor moral capaz de orientar nuestros actos. El abismo valorativo que así se establece hace que se llegue a preferir cualquier grado de sufrimiento animal a la lesión del más mínimo interés humano. Sería necesario, por tanto, reexaminar las situaciones de elección forzosa entre los intereses humanos y los animales para no dar preferencia más que a las opciones que encierran valores verdaderamente superiores. Sobre esta base podrían cuestionarse ciertas 
prácticas humanas habituales como: deportes o diversiones que conllevan sufrimiento animal, uso de los animales para adorno o vestido y, en general, cualquier uso para fines aparentemente secundarios. Los defensores más radicales de los animales llegan incluso a cuestionar la alimentación cárnica y la experimentación científica con animales(6).

\section{Relación entre bienestar animal y bioética}

Al abordar este aspecto, es indispensable acudir a algunas de las definiciones de "bienestar animal", incluidas en el manual del Consejo Canadiense para la Protección de los Animales CCPA(7): Broom lo define como "el estado en el cual se encuentra un animal que trata de adaptarse a su ambiente". La Asociación Mundial Veterinaria (AMV) afirma que la etología animal "pone el énfasis en el conocimiento científico. Su objetivo es de clarificar: a) las necesidades a satisfacer, y b) los daños que se pueden evitar". La American Veterinary Medical Association (AVMA) plantea que "todos los aspectos de bienestar animal incluyen el alojamiento apropiado, el manejo, la alimentación, el tratamiento y la prevención de enfermedades, el cuidado responsable, la manipulación humanitaria $\mathrm{y}$, cuando sea necesaria, la eutanasia humanitaria".

El bienestar animal, es el resultado de la interacción animal-ambiente, pero no el ambiente referido sólo a las condiciones del medio, sino un ambiente que incluye lo social. Además, el hecho de que los animales no tengan obligaciones morales no implica que los hombres puedan orientar su moralidad sin tener en cuenta a los animales. $\mathrm{O}$, dicho en otras palabras: que los animales no tengan deberes hacia nosotros, no implica que nosotros no los tengamos hacia ellos(6).

\section{Principios éticos específicos que aseguran el bienestar(8)}

1. Posibilitar el mínimo de manipulaciones al animal y las intervenciones en su entorno, evitando perturbarlo o provocarle reacciones de alerta o refugio.

2. Ofrecerle un entorno confortable y protegido en cuanto a agentes físicos, químicos y biológicos.

3. Lograr la seguridad del confinamiento, evitando su escape o fuga, la penetración de otros animales, la exposición a daños y la ausencia de peligros.

4. Las áreas de alojamiento de los animales deben ser específicas para este propósito y responder a los requerimientos establecidos, para la actividad de que se trate.

5. Lograr los objetivos del experimento, ensayo o validación con el mínimo de variables de tiempo y de animales.

Existen, por otra parte, una serie de principios aprobados por el Consejo de Organizaciones Internacionales de las Ciencias Médicas (CIOMS) que deben ser tomados en cuenta en cualquier investigación que se realice con diferentes animales de experimentación.

\section{Medidas que contribuyen a asegurar el bienestar(9)}

Para asegurar el bienestar de estos animales es imprescindible constatar:

- La estructura de la institución y de las áreas o departamentos donde se utilicen animales de laboratorio.

- Líneas de autoridad y responsabilidad para administrar y asegurar el cumplimiento de 
los programas de mantenimiento, cuidado y salud de animales.

- La calificación, autoridad y responsabilidad del (de los) veterinario(s) que participa(n) en los programas y el porcentaje de tiempo que aporta(n).

- La constitución de un Comité Institucional de Ética para el Cuidado y Uso de Animales de Laboratorio (CIECUAL).

- Los procedimientos que seguirá el CIECUAL para cumplir con los requisitos establecidos en el programa.

- El programa de salud para el personal que trabaja en las instalaciones de animales o que tiene contacto frecuente con éstos.

- El entrenamiento o la instrucción en el uso y cuidado de animales, así como la aplicación de métodos que reduzcan el número de animales requeridos para obtener resultados válidos o que limiten su sufrimiento.

- Las medidas de superficie de cada instalación, las especies alojadas y el porcentaje de inventario por especies.

Los principios éticos específicos y las medidas de aseguramiento, una vez aplicados, permiten brindar, tanto en la sala de alojamiento como en el área de encierro - que constituyen el ambiente con el cual se relaciona directamente el animal-, las condiciones que harán posible su bienestar.

Los factores presentes en el ambiente pueden ser de naturaleza física, química o biológica, los más comunes (algunos propios sólo de ambientes primarios) pueden $\operatorname{ser}(8)$ :

- Factores o agentes químicos: $\mathrm{NH}_{3}, \mathrm{CO}_{2}$, agentes naturales y artificiales.
- Físicos: temperatura, humedad relativa, ruido, iluminación, ventilación y cama.

- Biológicos: microorganismos, otros animales, densidad poblacional, patologías, genético.

\section{La selección de los biomodelos y su relación con el bienestar animal(10)}

La elección del biomodelo es un aspecto ético estrechamente relacionado con el bienestar animal. Un modelo es todo objeto de estudio que sirve de maqueta o prototipo del propio objeto, o de un objeto mayor. Es una copia, una imitación, una representación preliminar que sirve como plan y cuya finalidad es la construcción o formulación del objeto en estudio. El modelo puede ser inanimado -por ejemplo, un modelo matemático- o animado, cuando se trata de especies vegetales o animales, o sea "biomodelos". Éstos pueden ser inducidos o espontáneos, según el modo por el cual se obtuvieron; según el uso pueden ser clasificados en experimentales, para demostraciones (enseñanza), para ensayos o pruebas clínicas y para donantes de órganos o producción de biorreactivos.

En relación con la elección del mejor biomodelo, el investigador debe tomar en cuenta dos niveles:

- Individual: Selección cuidadosa de su biomodelo, con alto rigor ético y biológico, teniendo presente los principios éticos establecidos y reconocidos internacionalmente. Un principio aceptado por consenso establece empezar con sistemas no vivos (matemáticos, modelos de análisis y computación, realidad virtual), seguir con análisis químico y después pasar a los sistemas vivos como los métodos microbiológicos, el cultivo de células, tejidos y órganos, el uso de plantas e invertebrados, cuando sean 
apropiados y aceptados, en función de la disminución del número de los animales de experimentación o la sustitución de éstos. De este modo se sigue una ruta lógica para la selección, aunque muchas veces existe la tendencia a guiarse por lo que se encuentra en la literatura consultada, lo cual es válido para los casos ampliamente demostrados.

- Institucional: Comprende los procedimientos llevados a cabo por los llamados Comités Institucionales para el Cuidado y Uso de los Animales de Laboratorio, o también a través de los Comités de Ética para la Experimentación Animal.

\section{Los métodos alternativos $(3,11)$}

Restan muchos campos de investigación biomédica en los que, al menos en el futuro inmediato, se necesitará usar animales en los experimentos. Un animal vivo intacto es más que una suma de reacciones de células, tejidos u órganos independientes; existen complejas interacciones que los métodos alternativos biológicos o de otra índole no permiten duplicar, como en los casos donde se estudian fenómenos conductuales o de interacciones en el sistema nervioso. El término "alternativo" ha sido empleado para referirse a la sustitución de los animales vivos por otros procedimientos y a los métodos destinados a reducir el número de animales necesarios o a perfeccionar los procedimientos de experimentación.

Partiendo de estos criterios se toma el concepto de las tres $\mathrm{R}$, internacionalmente conocido, que expresa que en la experimentación animal debe preferirse toda técnica que:

- Reemplace el uso de animales o la sustitución por invertebrados, embriones de vertebrados, microorganismos, plantas, cultivos de células, tejidos u órganos.
- Reduzca la cantidad de animales empleados, sin perder la significación estadística de los resultados, logrando una menor dispersión de los datos, o que

- Refine un método existente para disminuir el dolor o malestar en los animales.

Lo que justifica el uso de métodos alternativos son los cuestionamientos éticos y las consideraciones prácticas, económicas y ecológicas. A pesar de ello, cualquiera técnica que se proponga requiere estar validada.

\section{EI dolor en los procedimientos de investigación. El punto final $(12,13)$}

Dar una definición del dolor en cada especie animal y determinar el valor umbral es una tarea sumamente compleja que requiere, por otra parte, provocar a un gran grupo de animales diversas intensidades de dolor.

La minimización y eliminación del dolor real o potencial y del diestrés en los animales debe estar presente, como parte de las técnicas de refinamiento, en cualquier actividad que implique su utilización. El diestrés es considerado un estado adverso, donde el animal es incapaz de adaptarse completamente al elemento estresante y, por lo tanto, muestra una conducta de inadaptación. Puede inducir cambios fisiológicos y psicológicos que no sólo afectan al animal sino también los resultados del experimento.

Muchos científicos usan tranquilizantes, analgésicos y anestésicos en los procesos de investigación con animales. Para ello se valen de tablas con las dosis específicas, según el tipo de fármaco y la especie en cuestión. Pero el asunto más álgido es determinar en qué momento detener una investigación para no ocasionar daño innecesario. Este momento se conoce como "punto final", en el cual 
se reduce, minimiza o termina el dolor o el diestrés del animal mediante la finalización del procedimiento, la administración de un tratamiento analgésico o la aplicación de eutanasia.

¿Qué observar en los animales para detectar diestrés? Morton y Griffings(14) sugieren poner atención a los cambios en la conducta espontánea, en el peso corporal (incluyendo cambios relacionados con el consumo de agua y alimento), en la apariencia física externa, en signos clínicos medibles (frecuencia cardiaca o respiratoria y su naturaleza) y en las respuestas conductuales a estímulos externos. Otros indicadores hormonales también son importantes, pero los anteriores parecen ser los más prácticos y accesibles. El investigador o el observador deben conocer las características conductuales de la especie observada.

Resulta indudable que los seres humanos tienen ciertas obligaciones morales en la utilización de animales de laboratorio. Existen cuatro deberes fundamentales, según SánchezGonzález(6):

- Definir los propósitos que pueden legitimar el uso de los animales de laboratorio.

- Ejercer un control sobre los niveles de dolor que se producen.

- Asegurar condiciones tolerables de alojamiento y cuidados.

- Mantener transparencia y responsabilidad públicas de los profesionales implicados.

Es preciso y urgente reconocer que toda forma de vida es un valor en sí mismo, que debe ser respetada y protegida. Los animales, al igual que el resto de los seres, tienen derecho a existir, con independencia de nosotros mismos y de aquellas conveniencias nuestras que no sean primordiales.
Dependemos de un ecosistema que está siendo alterado por las acciones del hombre, por lo que, al interactuar con los animales, estamos actuando sobre nosotros mismos. El respeto hacia los animales no es incompatible con el respeto hacia los seres humanos. Ambos forman parte de un único y más amplio "respeto hacia todo y hacia todos". Claro está que respetar no es no tocar: es valorar, comprender y estimar lo que se toca; $y$, sobre todo, hacerse responsable de todo lo que se toca(6).

El conocimiento debe seguir incrementándose y aplicándose en beneficio de toda la humanidad. El hombre de ciencia debe tener una estrecha vinculación con sus resultados, lo que abre un nuevo campo de responsabilidades con el avance científico-tecnológico.

Según expresa Núñez Jover(15), tanto a la ciencia como a los científicos no se les puede pedir sólo criterios cognoscitivos, sino también ideológicos y políticos. Todos los científicos tienen la obligación moral de tomar conciencia de su papel y responsabilidad social.

En Cuba, desde 1991, existe obligación de presentar documentación que respalde la aplicación de principios éticos en los ensayos clínicos (comités de ética, comités de revisión, entre otros). Se ha tomado conciencia en forma paulatina sobre la necesidad del comportamiento ético respecto de los animales de laboratorio. La introducción de las Buenas Prácticas de Laboratorio (BPL), la acreditación de laboratorios y exigencias de comportamiento dentro de la documentación del personal han contribuido a sentar las bases legales para el desarrollo de una cultura ética en el uso y cuidado de los animales de laboratorio. Más recientemente, la creación de la Sociedad de Animales de Laboratorio constituye un marco propicio para lograr uniformidad en el enfoque nacional del código de ética para el uso de estos animales(16). 


\section{Referencias}

1. Minteer BA, Collins JP. Ecological ethics: building a new tool kit for ecologists and biodiversity managers. Conservation Biology 2005; 19(6): 1803-1812.

2. ILAR, NRC. Guide for the Care and Use of Laboratory Animals. Washington, D.C.: National Academic Press; 1996.

3. Russell WMS, Burch RL. The Principles of Humane Experimental Technique en 1959 and The Three R's: The Way Forward. J. Zurlo, D. Rudacille, and A. M. Goldberg. Environmental Health Perspectives 1996; 104(8).

4. Capó M. Bioética animal: desarrollo de un concepto privado. Animales de Experimentación 1999; 4(5).

5. Núñez Jover J. La ciencia y la tecnología como procesos sociales. La Habana: Editorial Félix Varela; 1999.

6. Sánchez-González MA. La ética del uso de animales con fines científicos. Cuadernos del Programa Regional de Bioética 1997; 3.

7. CCPA. Manual, vol. 1 (2da edición), 1998: Capítulo VI.

8. González F, De la Peña R. Lineamientos Generales para la elaboración del Reglamento y las Normas de trabajo en los Vivarios Experimentales de los Institutos y Facultades de Ciencias Méd. La Habana: Centro Nac. de Perfeccionamiento Médico y Medios Audiovisuales, Minsap eds.; 1988.

9. De la Peña R. Problemas que afectan el Bienestar de los animales de laboratorio en procesos investigativos. I Forum Tecnológico Especial de la Ciencia de los Animales de Laboratorio. CENPALAB, 7-10 octubre; 2004.

10. Loew FM. Animals in Research. En Thomasma DC, Kushner T, (ed.) Birth to Death. Science and Bioethics. Cambridge: Cambridge University Press; 1996: 301-312.

11. Festing S. The Animal Research Debate. The Political Quarterly 2005; 76(4): 568-572.

12. Canadian Council on Animal Care. La selección del punto final apropiado en experimentos en que se utilizan animales para investigación cientifica, enseñanza y pruebas de laboratorio. 2000.

13. Conlee KM, Stephens ML, Rowan AN, King LA. Carbon dioxide for euthanasia: concerns regarding pain and distress, with special reference to mice and rats. Lab Anim 2005; 39(2): 137-161.

14. Morton DB, Griffiths PHM. Guidelines on the recognition of pain and discomfort in experimental animals and a hypothesis for assessment. Veterinary Record 1985; 116: 431-436.

15. Núñez Jover J. Tecnología y Sociedad. En: Problemas Sociales de la Ciencia y la Tecnología. La Habana: GESICIT, Editorial Félix Varela; 1994.

16. De la Torre A, Figueroa JM, Martínez L. El código de ética en la experimentación animal no puede ser letra muerta. Anuario Toxicología 2001; 1(1):140-145.

Recibido el 25 de febrero de 2007.

Aceptado el 15 de marzo de 2007. 